\title{
On New Generalized Fuzzy Directed Divergence Measure and Its Application in Decision Making Problem
}

\author{
Bhagwan Dass ${ }^{1, *}$, Vijay Prakash Tomar ${ }^{2}$, Krishan Kumar², Vikas Ranga ${ }^{3}$ \\ ${ }^{1}$ Department of Mathematics, Govt. College Jind -126102, India \\ ${ }^{2}$ Department of Mathematics, DCR University of Science and Technology, Murthal-131039, India \\ ${ }^{3}$ Department of Mathematics, Lovely Professional University, Punjab-144411, India
}

Received January 26, 2021; Revised March 18, 2021; Accepted April 18, 2021

\section{Cite This Paper in the following Citation Styles}

(a): [1] Bhagwan Dass, Vijay Prakash Tomar, Krishan Kumar, Vikas Ranga, "On New Generalized Fuzzy Directed Divergence Measure and Its Application in Decision Making Problem," Mathematics and Statistics, Vol. 9, No. 5, pp. 711 - 717, 2021. DOI: 10.13189/ms.2021.090510.

(b): Bhagwan Dass, Vijay Prakash Tomar, Krishan Kumar, Vikas Ranga (2021). On New Generalized Fuzzy Directed Divergence Measure and Its Application in Decision Making Problem. Mathematics and Statistics, 9(5), 711 - 717. DOI: 10.13189/ms.2021.090510.

Copyright $\odot 2021$ by authors, all rights reserved. Authors agree that this article remains permanently open access under the terms of the Creative Commons Attribution License 4.0 International License

\begin{abstract}
The concept of fuzzy sets presented by Zadeh has conquered an enormous achievement in numerous fields. Uncertainty in real world is ubiquitous. Entropy is an important tool with uncertainty and fuzziness. In this article, we propose new measure of directed divergence on fuzzy set. The extension of the fuzzy sets and one that integrated with other theories have been applied by some researchers. To prove the validity of measure, some axioms are proved. Using the proposed measure, we generate a method about decision making criteria and give a suitable method. In this article, we describe directed divergence measure for fuzzy set. Properties of proposed measure are discussed. In the real world, the multicriteria decision making is a very practical method and has a wide range of uses. By using multicriteria decision making, we can find best choice among the given criteria. In recent years, many researchers extensively apply fuzzy directed divergence for multicriteria decision making. Also some researchers defined the application of parameterized Hesitant Fuzzy Soft Set theory in decision making. In this article, we shall investigate the multiple criteria decision making problem under fuzzy environment. Application of introduced measure is given for decision making problem. A numerical example is given for decision making problem. In a fuzzy multicriteria problem, the analysis is given by an illustration example of the new
\end{abstract}

define approach regarding admission preference of a student for post graduate course of science stream.

Keywords Fuzzy Set, Divergence Measure, Directed Divergence, Decision Making

\section{Introduction}

The study of problem concerning which includes information, dispensation, storage retrieval and decision making are dealt with the help of information theory and fuzzy theory. First time Shannon [1] gives an idea that the measure of information theory is termed as entropy. After that Kullback and Liebler [2] evaluate that measure of information associated with the two probability distribution $p_{i}$ and $q_{i}$ of discrete random variable, is given as

$$
D\left(p / / q_{i}\right)=\sum_{i=1}^{n} p_{i} \log \frac{p_{i}}{q_{i}}
$$

known as directed divergence. L. Zadeh [3] introduced the fuzzy set theory and the concept of fuzzy set theory is used in different areas of science and technology e.g. image 
processing, pattern recognition, decision making etc. A fuzzy distance measure between two fuzzy set was proposed by Bhandari and Pal [4] using the concept of fuzzy measure conditioning corresponding to Kullbck and Leibler [2] probabilistic directed divergence. Bhandari and $\mathrm{Pal}$ [4] introduced a measure

$$
\begin{aligned}
& I(A, B)=\sum_{i=1}^{n} \mu_{A}\left(x_{i}\right) \log \frac{\mu_{A}\left(x_{i}\right)}{\mu_{B}\left(x_{i}\right)}+ \\
& +\left(1-\mu_{A}\left(x_{i}\right)\right) \log \frac{\left(1-\mu_{A}\left(x_{i}\right)\right)}{\left(1-\mu_{B}\left(x_{i}\right)\right)}
\end{aligned}
$$

Later, Fan and Xie [5] proposed discrimination of fuzzy information of fuzzy set $A$ against $B$, corresponding to exponential fuzzy entropy given by Pal and Pal [6]. Kapur [7] introduced a generalized directed divergence measure corresponding to Havrda and Charvat [8]. Hooda and Bajaj [9] proposed directed divergence measure along with R-norm directed divergence. Bhatia and Singh [10] gave some measure of directed divergence of two set $A$ and $B$. Tomar and Ohlan and Priya and Tomar [11, 14] proposed some measure of fuzzy directed divergence. Zahari Md Rodzi, Abd Ghafur Ahmad [15] defined the application of parameterized Hesitant Fuzzy Soft Set theory in decision making. Keeping in mind above literature we propose directed divergence and some important properties are also studied. It is shown that propose measure has wide application in pattern. In section IInd, a brief study about fuzzy set, measure and directed divergence is given. In section IIIrd, a new directed divergence measure is discussed. In section IVth, properties are described with their proof. Application of proposed measure is discussed in section Vth. At last, the conclusion of all above work is given in VIth section.

\section{Preliminaries}

In this section we define some definitions and notations about fuzzy set and directed divergence measure. We will present those aspects of fuzzy set and its measure which will be used in our next discussion.

Definition 1. Let $\Gamma_{n}=\left\{P=\left(p_{1}, p_{2}, \ldots \ldots p_{n}\right) / p_{i} \geq 0\right\}$ is the set of all complete finite discrete probability distribution then measure of information was defined firstly by Shannon as

$$
H(P)=\sum_{i=1}^{n} p_{i} \log p_{i}, \quad P \in \Gamma_{n}
$$

Definition 2. Let $X=\left\{x_{1}, x_{2}, \ldots \ldots x_{n}\right\}$ be universe of discourse then $A=\left\{\prec x, \mu_{A}\left(x_{i}\right) \succ / x \in X\right\}$ is called fuzzy set where $\mu_{A}\left(x_{i}\right): X \rightarrow[0,1]$ is a membership function defined as follows

$$
\mu_{A}\left(x_{i}\right)=\left\{\begin{array}{rll} 
& 0 & \text { if } x \notin A \\
& 1 & \text { if } x \in A \\
0.5 & \text { if } & x \notin A \text { or } x \in A
\end{array}\right\}
$$

Some notation for two fuzzy set

$$
\begin{array}{ll}
\text { 1. } & A \cup B=\left\{\prec x, \max \left(\mu_{A}(x), \mu_{B}(x)\right) \succ / x \in X\right\} \\
\text { 2. } & A \cap B=\left\{\prec x, \min \left(\mu_{A}(x), \mu_{B}(x)\right) \succ / x \in X\right\} \\
\text { 3. } & A=B=\left\{\prec x, \mu_{A}(x)=\mu_{B}(x) \succ / x \in X\right\} \\
\text { 4. } & A . B=\left\{\prec x, \mu_{A}(x) \cdot \mu_{B}(x) \succ / x \in X\right\} \\
\text { 5. } & A^{C}=\left\{\prec x, \mu_{A}(x)=1-\mu_{A}(x) \succ / x \in X\right\}
\end{array}
$$

Definition 3. Let $X=\left\{x_{1}, x_{2}, \ldots \ldots x_{n}\right\}$ be universe of discourse and $F(X)$ be the set of all family subset. A mapping $I: F(X) \times F(X) \rightarrow R$ is called divergence measure between fuzzy sets if

$$
\begin{array}{cl}
\text { i. } & I(A: B) \geq 0 \\
\text { ii. } & I(A: B)=I(B: A) \\
\text { iii. } & I(A: B)=0 \text { iff } A=B \\
\text { iv. } & \operatorname{Max}\{I(A \cup C, B \cup C), I(A \cap C, B \cap C)\} \leq I(A: B)
\end{array}
$$


Bajaj et.al.[12] defined the measure of fuzzy directed divergence as

$$
\begin{gathered}
I_{\alpha,}(A: B)=\frac{1}{\alpha-1} \sum_{i=1}^{n} \log \left[\mu_{A}^{\alpha}\left(x_{i}\right) \mu_{B}^{1-\alpha}\left(x_{i}\right)+\left(1-\mu_{A}\left(x_{i}\right)\right)^{\alpha}\left(1-\mu_{B}\left(x_{i}\right)\right)^{1-\alpha}\right] \\
I_{\alpha, \beta}(A: B)=\frac{1}{1-2^{\beta-1}} \sum_{i=1}^{n}\left(\left[\mu_{A}^{\alpha}\left(x_{i}\right) \mu_{B}^{1-\alpha}\left(x_{i}\right)+\left(1-\mu_{A}\left(x_{i}\right)\right)^{\alpha}\left(1-\mu_{B}\left(x_{i}\right)\right)^{1-\alpha}\right]^{\beta-1}-1\right)
\end{gathered}
$$

Prakash et. al. [13] introduced entropy measure on fuzzy set as

$$
H_{\alpha}^{\beta}(A)=\frac{1}{(1-\alpha) \beta} \sum_{i=1}^{n}\left(\left[\mu_{A}^{\alpha}\left(x_{i}\right)+\left(1-\mu_{A}\left(x_{i}\right)\right)^{\alpha}\right]^{\beta}-1\right) ; \alpha>0, \alpha \neq 1, \beta \neq 0
$$

\section{New Directed Divergence Measure}

Corresponding to Prakash et. al. [13] we propose the measure of fuzzy directed divergence as follow

$$
\left.H_{\alpha, \beta}(A, B)=\frac{1}{(\alpha-1) \beta} \sum_{i=1}^{n}\left(\begin{array}{l}
\mu_{A}^{\alpha}\left(x_{i}\right) \mu_{B}^{1-\alpha}\left(x_{i}\right) \\
+\left(1-\mu_{A}\left(x_{i}\right)\right)^{\alpha}\left(1-\mu_{B}\left(x_{i}\right)\right)^{1-\alpha}
\end{array}\right]^{\beta}-1\right) ; \alpha>0, \alpha \neq 1, \beta \neq 0
$$

Theorem1. Show that $H_{\alpha, \beta}(A, B)$ is valid measure of fuzzy directed divergence.

Proof. To show that proposed measure in (1) is valid we have to prove following axioms

I. We can clearly check in figure that $H_{\alpha, \beta}(A, B)$ is non-negative.

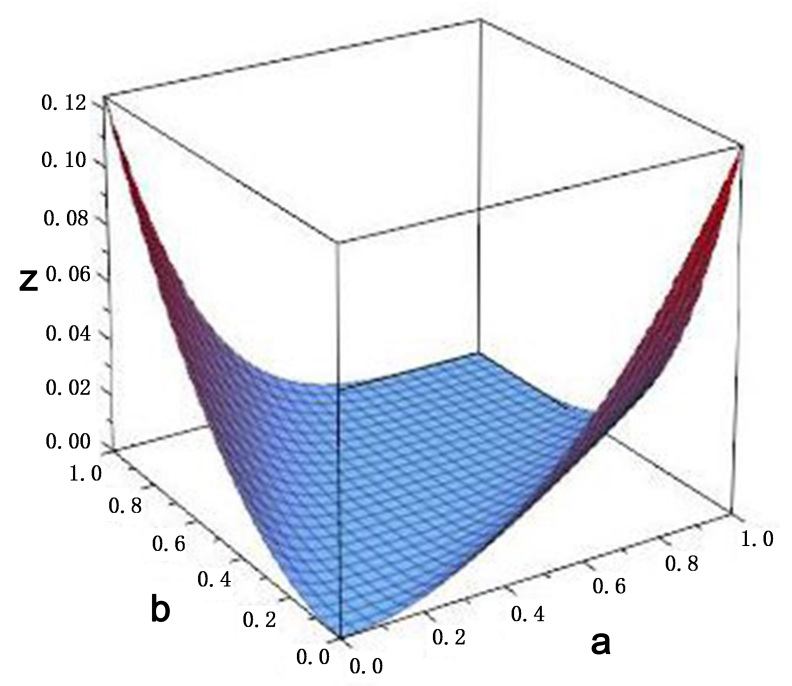

Figure 1. $H_{\alpha, \beta}(A, B)$

II. $\quad H_{\alpha, \beta}(A, B) \neq H_{\alpha, \beta}(B, A)$

But we know that $J_{\alpha, \beta}(A, B)=H_{\alpha, \beta}(A, B)+H_{\alpha, \beta}(B, A)$ is symmetric.

III. $\quad H_{\alpha, \beta}(A, B)=0$ if $A=B$

IV. We have to check the convexity of $H_{\alpha, \beta}(A, B)$.

So now,

$$
\frac{\partial H_{\alpha, \beta}}{\partial \mu_{A}\left(x_{i}\right)}=\left\{\alpha \beta\left[\begin{array}{l}
\mu_{A}^{\alpha-1}\left(x_{i}\right) \mu_{B}^{1-\alpha}\left(x_{i}\right) \\
+\left(1-\mu_{A}\left(x_{i}\right)\right)^{\alpha-1}\left(1-\mu_{B}\left(x_{i}\right)\right)^{1-\alpha}
\end{array}\right]\left[\begin{array}{l}
\mu_{A}^{\alpha}\left(x_{i}\right) \mu_{B}^{1-\alpha}\left(x_{i}\right) \\
+\left(1-\mu_{A}\left(x_{i}\right)\right)^{\alpha}\left(1-\mu_{B}\left(x_{i}\right)\right)^{1-\alpha}
\end{array}\right]^{\beta-1}\right\}
$$




$$
\begin{aligned}
& \frac{\partial^{2} H_{\alpha, \beta}}{\partial \mu_{A}^{2}\left(x_{i}\right)}=\left\{\begin{array}{l}
\alpha(\alpha-1) \beta\left[\mu_{A}^{\alpha-2}\left(x_{i}\right) \mu_{B}^{1-\alpha}\left(x_{i}\right)+\left(1-\mu_{A}\left(x_{i}\right)\right)^{\alpha-2}\left(1-\mu_{B}\left(x_{i}\right)\right)^{1-\alpha}\right] \\
{\left[\mu_{A}^{\alpha}\left(x_{i}\right) \mu_{B}^{1-\alpha}\left(x_{i}\right)+\left(1-\mu_{A}\left(x_{i}\right)\right)^{\alpha}\left(1-\mu_{B}\left(x_{i}\right)\right)^{1-\alpha}\right]^{\beta-1}} \\
+\alpha(\beta-1) \beta\left[\mu_{A}^{\alpha-1}\left(x_{i}\right) \mu_{B}^{1-\alpha}\left(x_{i}\right)+\left(1-\mu_{A}\left(x_{i}\right)\right)^{\alpha-1}\left(1-\mu_{B}\left(x_{i}\right)\right)^{1-\alpha}\right] \\
{\left[\mu_{A}^{\alpha}\left(x_{i}\right) \mu_{B}^{1-\alpha}\left(x_{i}\right)+\left(1-\mu_{A}\left(x_{i}\right)\right)^{\alpha}\left(1-\mu_{B}\left(x_{i}\right)\right)^{1-\alpha}\right]^{\beta-2}}
\end{array}\right\} \\
& \Rightarrow \quad \frac{\partial^{2} H_{\alpha, \beta}}{\partial \mu_{A}^{2}\left(x_{i}\right)}>0 \text { for } \alpha>0, \beta>0, \alpha \neq 1, \beta \neq 1,2 \text {, }
\end{aligned}
$$

Similarly we can show that $\Rightarrow \quad \frac{\partial^{2} H_{\alpha, \beta}}{\partial \mu_{B}^{2}\left(x_{i}\right)}>0$ for $\alpha>0, \beta>0, \alpha \neq 1,2, \beta \neq 1$,

So axiomatically it is clear that proposed measure are valid.

\section{Some Important Properties}

Assume that the family of all fuzzy set of universe $X$, is denote by $F S(X)$ and $A, B, C \in F S(X)$ is given

$$
\begin{aligned}
A & =\left[\prec x, \mu_{A}(x) \succ / x \in X\right] \\
B & =\left[\prec x, \mu_{B}(x) \succ / x \in X\right] \\
C & =\left[\prec x, \mu_{C}(x) \succ / x \in X\right]
\end{aligned}
$$

and we have $\Delta_{1}=\left[x_{i} / x_{i} \in X, \mu_{A}\left(x_{i}\right) \geq \mu_{B}\left(x_{i}\right)\right]$

$$
\Delta_{2}=\left[x_{i} / x_{i} \in X, \mu_{A}\left(x_{i}\right)<\mu_{B}\left(x_{i}\right)\right]
$$

Theorem2. Prove that proposed measure in (1) satisfies the following properties:

$$
\text { I. } \quad H_{\alpha, \beta}(A \cup B, A)+H_{\alpha, \beta}(A \cap B, A)=H_{\alpha, \beta}(B, A)
$$

II. $\quad H_{\alpha, \beta}(A, A \cap B)=H_{\alpha, \beta}(A \cup B, B)$

III. $\quad H_{\alpha, \beta}(A, A \cup B)=H_{\alpha, \beta}(A \cap B, B)$

IV. $\quad H_{\alpha, \beta}(A \cup B, C)+H_{\alpha, \beta}(A \cap B, C)=H_{\alpha, \beta}(A, C)+H_{\alpha, \beta}(B, C)$

V. $\quad H_{\alpha, \beta}(A \cup B, A \cap B)=H_{\alpha, \beta}(A \cup B, B)+H_{\alpha, \beta}(B, A \cap B)$

VI. $\quad H_{\alpha, \beta}\left(A, A^{C}\right)=H_{\alpha, \beta}\left(A^{C}, A\right)$

VII. $\quad H_{\alpha, \beta}\left(A^{C}, B^{C}\right)=H_{\alpha, \beta}(A, B)$

VIII. $\quad H_{\alpha, \beta}\left(A, B^{C}\right)=H_{\alpha, \beta}\left(A^{C}, B\right)$

IX. $\quad H_{\alpha, \beta}(A, B)+H_{\alpha, \beta}\left(A^{C}, B\right)=H_{\alpha, \beta}\left(A^{C}, B^{C}\right)+H_{\alpha, \beta}\left(A, B^{C}\right)$

Proof.

$$
\begin{aligned}
& {\left[\begin{array}{l}
H_{\alpha, \beta}(A \cup B, A) \\
+H_{\alpha, \beta}(A \cap B, A)
\end{array}\right]=\frac{1}{(\alpha-1) \beta} \sum_{i=1}^{n}\left(\begin{array}{l}
{\left[\begin{array}{l}
\mu_{A \cup B}^{\alpha}\left(x_{i}\right) \mu_{A}^{1-\alpha}\left(x_{i}\right) \\
+\left(1-\mu_{A \cup B}\left(x_{i}\right)\right)^{\alpha}\left(1-\mu_{A}\left(x_{i}\right)\right)^{1-\alpha}
\end{array}\right]^{\beta}} \\
+\left[\begin{array}{l}
\mu_{A \cap B}^{\alpha}\left(x_{i}\right) \mu_{A}^{1-\alpha}\left(x_{i}\right) \\
+\left(1-\mu_{A \cap B}\left(x_{i}\right)\right)^{\alpha}\left(1-\mu_{A}\left(x_{i}\right)\right)^{1-\alpha}
\end{array}\right]^{\beta}-2
\end{array}\right)}
\end{aligned}
$$

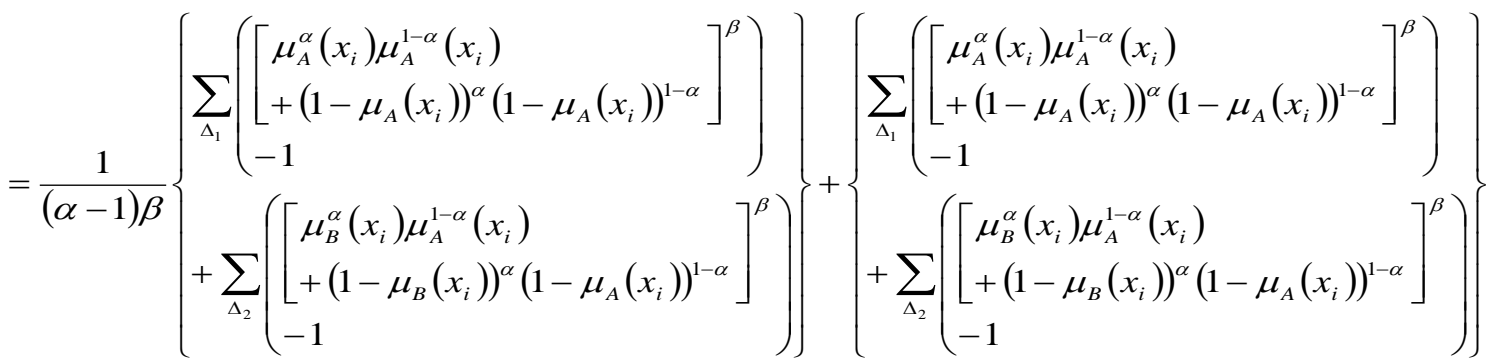




$$
=\frac{1}{(\alpha-1) \beta} \sum_{i=1}^{n}\left(\left[\begin{array}{l}
\mu_{B}^{\alpha}\left(x_{i}\right) \mu_{A}^{1-\alpha}\left(x_{i}\right) \\
+\left(1-\mu_{B}\left(x_{i}\right)\right)^{\alpha}\left(1-\mu_{A}\left(x_{i}\right)\right)^{1-\alpha}
\end{array}\right]^{\beta}-1\right)
$$

Hence we can say that

$$
H_{\alpha, \beta}(A \cup B, A)+H_{\alpha, \beta}(A \cap B, A)=H_{\alpha, \beta}(B, A)
$$

II. $\quad H_{\alpha, \beta}(A, A \cap B)=\frac{1}{(\alpha-1) \beta} \sum_{i=1}^{n}\left(\left[\begin{array}{l}\mu_{A}^{\alpha}\left(x_{i}\right) \mu_{A \cap B}^{1-\alpha}\left(x_{i}\right) \\ +\left(1-\mu_{A}\left(x_{i}\right)\right)^{\alpha}\left(1-\mu_{A \cap B}\left(x_{i}\right)\right)^{1-\alpha}\end{array}\right]^{\beta}-1\right)$

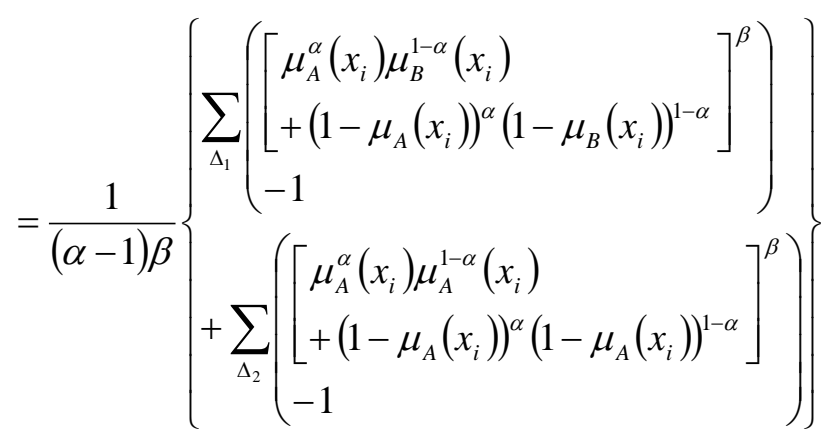

$$
\begin{aligned}
& \left.=\frac{1}{(\alpha-1) \beta}\left\{\sum_{\Delta_{1}}\left(\begin{array}{l}
\mu_{A}^{\alpha}\left(x_{i}\right) \mu_{B}^{1-\alpha}\left(x_{i}\right) \\
+\left(1-\mu_{A}\left(x_{i}\right)\right)^{\alpha}\left(1-\mu_{B}\left(x_{i}\right)\right)^{1-\alpha} \\
-1
\end{array}\right]^{\beta}\right)\right\} \\
& \left.H_{\alpha, \beta}(A \cup B, B)=\frac{1}{(\alpha-1) \beta} \sum_{i=1}^{n}\left(\begin{array}{l}
\mu_{A \cup B}^{\alpha}\left(x_{i}\right) \mu_{B}^{1-\alpha}\left(x_{i}\right) \\
+\left(1-\mu_{A \cup B}\left(x_{i}\right)\right)^{\alpha}\left(1-\mu_{B}\left(x_{i}\right)\right)^{1-\alpha}
\end{array}\right]^{\beta}-1\right) \\
& =\frac{1}{(\alpha-1) \beta}\left\{\begin{array}{l}
\sum_{\Delta_{1}}\left(\begin{array}{l}
{\left[\begin{array}{l}
\mu_{A}^{\alpha}\left(x_{i}\right) \mu_{B}^{1-\alpha}\left(x_{i}\right) \\
+\left(1-\mu_{A}\left(x_{i}\right)\right)^{\alpha}\left(1-\mu_{B}\left(x_{i}\right)\right)^{1-\alpha}
\end{array}\right]^{\beta}} \\
-1
\end{array}\right]^{\beta}\left\{\begin{array}{l}
{\left[\begin{array}{l}
\mu_{B}^{\alpha}\left(x_{i}\right) \mu_{B}^{1-\alpha}\left(x_{i}\right) \\
+\left(1-\mu_{B}\left(x_{i}\right)\right)^{\alpha}\left(1-\mu_{B}\left(x_{i}\right)\right)^{1-\alpha}
\end{array}\right]^{\beta}} \\
+1
\end{array}\right) \\
+\sum_{\Delta_{2}}
\end{array}\right\} \\
& \left.=\frac{1}{(\alpha-1) \beta}\left\{\sum_{\Delta_{1}}\left(\begin{array}{l}
\mu_{A}^{\alpha}\left(x_{i}\right) \mu_{B}^{1-\alpha}\left(x_{i}\right) \\
+\left(1-\mu_{A}\left(x_{i}\right)\right)^{\alpha}\left(1-\mu_{B}\left(x_{i}\right)\right)^{1-\alpha}
\end{array}\right]^{\beta}\right)\right\}
\end{aligned}
$$

Hence we can say that

$$
H_{\alpha, \beta}(A, A \cap B)=H_{\alpha, \beta}(A \cup B, B) .
$$

All other properties can be proved as above. 


\section{Application of Directed Divergence Measure in Decision Making}

In the real world, the multicriteria decision making is a very practical method and has wide use. By using multicriteria decision making we can find best choice among the given criteria. In recent years, many researchers extensively apply fuzzy directed divergence for multicriteria decision making. In this article we shall investigate the multiple criteria decision making problem under fuzzy environment. For multicriteria decision making problem, we have a set of strategies say $A_{1}, A_{2}, A_{3}, \ldots \ldots \ldots \ldots A_{n}$ and suppose that each strategy has varied degree of effectiveness w. r. t. cost set $C_{1}, C_{2}, C_{3}, \ldots \ldots \ldots \ldots C_{n}$.

Step -1. First we arrange the preference of decision makers in the form of fuzzy decision making matrix for each alternative $A_{j}(j=1,2, \ldots \ldots . n)$ w. r. t. cost set $C_{k}(k=1,2, \ldots \ldots . m)$ as follows

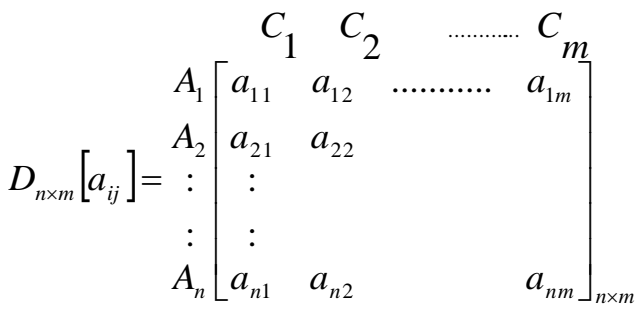

Step-2. We determine the ideal solution $A^{*}$ from all alternative corresponding to their cost set as

$$
A^{*}=\left\{H_{1}^{*}, H_{2}^{*}, \ldots \ldots \ldots H_{n}^{*}\right\} \text { where } H_{l}^{*}=\max \left\{H_{i}^{*}\right\}
$$

Step -3. Therefore we calculate the divergence using $H_{\alpha, \beta}(A: B)$ given as

$$
\left.H_{\alpha, \beta}(A, B)=\frac{1}{(\alpha-1) \beta} \sum_{i=1}^{n}\left(\begin{array}{l}
\mu_{A}^{\alpha}\left(x_{i}\right) \mu_{B}^{1-\alpha}\left(x_{i}\right) \\
+\left(1-\mu_{A}\left(x_{i}\right)\right)^{\alpha}\left(1-\mu_{B}\left(x_{i}\right)\right)^{1-\alpha}
\end{array}\right]^{\beta}-1\right)
$$

Step -4. To give ranking we take

$$
\min \left\{H_{\alpha, \beta}\left(A_{j}, A^{*}\right)\right\} ; \text { where } 1 \leq j \leq n
$$

Select the most desirable alternative according with descending order of their function.

\section{Numerical Example}

In a fuzzy multicriteria problem we analyze an illustration example of the new define approach regarding admission preference of a student for post graduate course of science stream. Suppose that the student wants to take admission in Indian Institute of Technology (IIT) and he wants to select an institute from five options

$$
\begin{aligned}
& A_{1}=\text { IIT Delhi } \\
& A_{2}=\text { IIT Bombay } \\
& A_{3}=\text { IIT Madras } \\
& A_{4}=\text { IIT Kanpur } \\
& A_{5}=\text { IIT Roorki }
\end{aligned}
$$

These are the most valuable institute for science course. The student wants to choose institute on the following basis

$$
\begin{aligned}
& C_{1}=\text { Placement } \\
& C_{2}=\text { Ranking } \\
& C_{3}=\text { Faculty } \\
& C_{4}=\text { Facility } \\
& C_{5}=\text { Fee }
\end{aligned}
$$

Step-1. Arranging the value of all alternative we arrange the preference in matrix $M_{n \times m}\left\lfloor m_{i j}\right\rfloor$.

$$
\begin{aligned}
& \begin{array}{llllll}
C_{1} & C_{2} & C_{3} & C_{4} & C_{5}
\end{array} \\
& A_{1}\left[\begin{array}{lllll}
0.5 & 0.7 & 0.8 & 0.3 & 0.1
\end{array}\right] \\
& \begin{array}{l|lllll}
A_{2} & 0.7 & 0.9 & 0.3 & 0.2 & 0.4
\end{array} \\
& M_{n \times m}\left[m_{i j}\right]=A_{3} \quad 0.4 \quad 0.8 \quad 0.6 \quad 0.7 \quad 0.5 \\
& \begin{array}{l|lllll}
A_{4} & 0.3 & 0.1 & 0.5 & 0.4 & 0.2
\end{array} \\
& A_{5}\left[\begin{array}{lllll}
0.1 & 0.2 & 0.4 & 0.8 & 0.6
\end{array}\right]_{n \times m}
\end{aligned}
$$

Step-2. Optimum solution from above matrix is

$$
A^{*}=\{0.7,0.9,0.8,0.8,0.6\}
$$

Step-3. We calculate the divergence of $A^{*}$ w. r. t. each alternative as

Table1. Different values of entropy at different parameters

\begin{tabular}{|c|c|c|c|c|c|c|}
\hline$\alpha$ & $\beta$ & $H_{\alpha, \beta}\left(A_{1}, A^{*}\right)$ & $H_{\alpha, \beta}\left(A_{2}, A^{*}\right)$ & $H_{\alpha, \beta}\left(A_{3}, A^{*}\right)$ & $H_{\alpha, \beta}\left(A_{4}, A^{*}\right)$ & $H_{\alpha, \beta}\left(A_{5}, A^{*}\right)$ \\
\hline 0.1 & 0.1 & 0.14 & 0.14 & 0.03 & 0.31 & 0.25 \\
\hline 0.9 & 0.1 & 1.25 & 1.36 & 0.34 & 2.81 & 2.33 \\
\hline 1.1 & 2.1 & 1.56 & 1.74 & 0.43 & 3.68 & 3.05 \\
\hline 5 & 2.1 & 30.25 & 72.54 & 2.60 & 1960.1 & 960.5 \\
\hline
\end{tabular}


Step- 4. From the table-1 we find that $H_{\alpha, \beta}\left(A_{3}, A^{*}\right)$ has minimum value for all parameters so we can easily estimate that the best alternative is $A_{3}$. So the student should take admission in IIT Madras.

Hence this shows that introduced fuzzy measure divergence is very suitable measure to solve the multicriteria decision making problem.

\section{Conclusions}

In this article we describe directed divergence measure for fuzzy set. Properties of proposed measure are discussed. Application of introduced measure is given for decision making. A numerical example is given for decision making.

\section{Conflict of Interest}

There is no any conflict of interest.

\section{Ethical Approval}

In present article, the authors have no any study with human participants or animals.

\section{REFERENCES}

[1] Shannon E (1965) A Mathematical Theory of Communication. The Bell System Technical Journal, 27:379-423.

[2] Kullback S and Leibler RA (1952) On information and sufficiency. Ann. Math. Stat., 22: 79 - 86.

[3] Zadeh LA (1965) Fuzzy Sets. Information and Control, 8:
338-353.

[4] Bhandari D and Pal NR (1993) Some new information measures for fuzzy sets. Information Science, 67: 204 - 228.

[5] Fan J and Xie W (1999) Distance Measures and Induced Fuzzy Entropy. Fuzzy Sets and Systems, 104(2):305-314.

[6] Pal NR and Pal SK (1999) Entropy: a new definitions and its applications. IEEE Transactions on systems, Man and Cybernetics, 21(5): 1260-1270.

[7] Kapur JN (1997) Measures of Fuzzy Information. Mathematical Sciences Trust Society, New Delhi, India.

[8] Havrda JH and Charvat F (1967) Quantification Methods of Classification Processed: Concepts of Structural Entropy. Kybernetika, 3:30-35.

[9] Hooda DS and Bajaj RK (2008) On generalized norm information measures of fuzzy information. Journal of the Applied Mathematics, Statistics and Informatics, 4(2):199-212.

[10] Bhatia PK and Singh S (2012) Three Families of Generalized Fuzzy Directed divergence. AMO-Advanced Modeling and Optimization, 14(3):599-614.

[11] Tomar VP and Ohlan A (2014) Sequence of fuzzy divergence measures and inequalities. AMO - Advanced Modeling and Optimization, 16(2):439-452.

[12] Bajaj RK and Hooda DS (2010) On some new generalized measures of fuzzy information. World Academy of Science, Engineering and Technology, 62:747-753

[13] Parkash O (1998) A new parametric measure of fuzzy entropy. Information Processing and Management of Uncertainty, 2:1732-1737.

[14] Priya Arora, V. P. Tomar , "Measuring Given Partial Information about Intuitionistic Fuzzy Sets," Mathematics and Statistics, Vol. 8, No. 6, pp. 665 - 670, 2020, DOI: 10.13189/ms.2020.080606.

[15] Zahari Md Rodzi, Abd Ghafur Ahmad, “ Application of Parameterized Hesitant Fuzzy Soft Set Theory in Decision Making" Mathematics and Statistics, Vol. 8, No. 3, pp. 244 - 253, 2020, DOI: 10.13189/ms.2020.080302. 